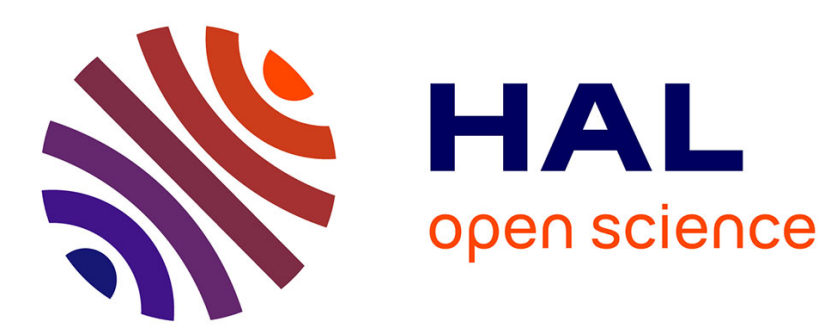

\title{
Effets d'anisotropie par homogénéisation dans un problème à frontière libre
}

Guy Bayada, Sébastien Martin, Carlos Vazquez

\section{To cite this version:}

Guy Bayada, Sébastien Martin, Carlos Vazquez. Effets d'anisotropie par homogénéisation dans un problème à frontière libre. 2004. hal-00085966

\section{HAL Id: hal-00085966 https://hal.science/hal-00085966}

Preprint submitted on 17 Jul 2006

HAL is a multi-disciplinary open access archive for the deposit and dissemination of scientific research documents, whether they are published or not. The documents may come from teaching and research institutions in France or abroad, or from public or private research centers.
L'archive ouverte pluridisciplinaire HAL, est destinée au dépôt et à la diffusion de documents scientifiques de niveau recherche, publiés ou non, émanant des établissements d'enseignement et de recherche français ou étrangers, des laboratoires publics ou privés. 


\title{
Anisotropic effects by homogenization in a free boundary problem
}

\author{
Effets d'anisotropie par homogénéisation dans un \\ problème à frontière libre \\ Guy Bayada ${ }^{\mathrm{a}, \mathrm{b}}$ Sébastien Martin ${ }^{\mathrm{a}}$ Carlos Vazquez ${ }^{\mathrm{c}}$ \\ a MAPLY CNRS-UMR 5585, INSA Lyon, Bât. Léonard de Vinci, F-69621 Villeurbanne Cedex, France. \\ ${ }^{\mathrm{b}}$ LAMCOS CNRS-UMR 5514, INSA Lyon, Bât. D'Alembert, F-69621 Villeurbanne Cedex, France. \\ ${ }^{\mathrm{c}}$ Department of Mathematics, Faculty of Informatics, University of A Coruña, Campus Elviña, 15071-A Coruña, Spain
}

\begin{abstract}
The Elrod-Adams model is a pressure-saturation formulation which takes into account cavitation phenomena in thin fluid films mechanics. We study the asymptotic behavior of the model, using the two-scale convergence technique, in devices such as journal bearings. Interest is highly motivated by the roughness effects of the surfaces. Anisotropic effects appear on the coefficients, which is usual, but also on the saturation function, since various saturation functions appear through the homogenization process.
\end{abstract}

\section{Résumé}

Le modèle d'Elrod-Adams est une formulation en pression-saturation, qui permet de prendre en compte les phénomènes de cavitation en mécanique des films minces. Nous étudions le comportement asymptotique, par homogénéisation double-échelle, de la solution dans des mécanismes lubrifiés de type coussinet, dont l'intérêt est motivé par la prise en compte des rugosités des surfaces. Les effets d'anisotropie apparaissent sur les coefficients, ce qui est classique, mais aussi sur la saturation.

Mots-clés : lubrification; cavitation ; frontière libre; homogénéisation double-échelle ; anisotropie

\section{Abridged English version}

We consider a bearing involving two rigid surfaces. The space between the surfaces is very thin and filled with some lubricant. In order to take into account cavitation phenomena, we use the Elrod-Adams model, which introduces a saturation function in the right-hand side of the Reynolds equation:

Email addresses: guy.bayada@insa-lyon.fr, [Tel./Fax. : +33472438312 / 85 29] (Guy Bayada), sebastien.martin@insa-lyon.fr (Sébastien Martin), carlosv@udc.es (Carlos Vazquez). 


$$
-\nabla \cdot\left(h^{3} \nabla p\right)=-\frac{\partial}{\partial x_{1}}(\theta h), \quad p \geq 0, \quad H(p) \leq \theta \leq 1 .
$$

The gap $h$ is the normalized distance between the two surfaces, $(p, \theta)$ is the pressure-saturation, and $H$ is the Heaviside graph. Actually, the saturation denotes the local ratio of liquid fluid between the two surfaces, so that a free boundary separates non-cavitated areas (in which $p>0$ and $\theta=1$ ) from cavitated areas (in which $p=0$ and $0 \leq \theta \leq 1$ ). The weak formulation is given by:

$$
(\mathcal{P})\left\{\begin{array}{l}
\text { Find }(p, \theta) \in V_{a} \times L^{\infty}(\Omega) \text { such that } \\
\int_{\Omega} h^{3} \nabla p \cdot \nabla v d x=\int_{\Omega} \theta h \frac{\partial v}{\partial x_{1}} d x, \quad \forall v \in V_{0}, \\
p \geq 0, \quad H(p) \leq \theta \leq 1 \quad \text { a.e. in } \Omega,
\end{array}\right.
$$

the functional spaces being defined as

$$
\begin{aligned}
& V_{a}=\left\{v \in H^{1}(\Omega), v \text { is } 2 \pi x_{1} \text {-periodic, } v_{\left.\right|_{\Gamma_{0}}}=0, v_{\left.\right|_{\Gamma_{a}}}=p_{a}\right\}, \\
& V_{0}=\left\{v \in H^{1}(\Omega), v \text { is } 2 \pi x_{1} \text {-periodic, } v_{\left.\right|_{\Gamma_{0}}}=0, v_{\left.\right|_{\Gamma_{a}}}=0\right\},
\end{aligned}
$$

with $\Omega=] 0,2 \pi[\times] 0,1\left[, \Gamma_{0}=\right] 0,2 \pi\left[\times\{0\}, \Gamma_{a}=\right] 0,2 \pi\left[\times\{1\}\right.$ and $p_{a}>0 . h$ is a regular bounded function which is greater than a strictly positive constant. From the techniques used in $[4,5]$, we prove:

Theorem 0.1 Problem $(\mathcal{P})$ admits at least one solution $(p, \theta)$. Moreover, the pressure $p$ is unique, and if there exists a set of positive measure such that $p\left(x_{1}, x_{2}\right)>0$, for almost every $\left.x_{2} \in\right] 0,1[$, then the saturation $\theta$ is unique. In particular, if $h$ can be written as $h\left(x_{1}, x_{2}\right)=h_{1}\left(x_{1}\right) h_{2}\left(x_{2}\right)$ (where $h_{i}$ is a regular bounded function, greater than a strictly positive constant), problem $(\mathcal{P})$ admits a unique solution.

The homogenization study is motivated by the roughness of the surfaces due to the manufacturing process. Thus the effective gap, denoted $h_{\varepsilon}(x)=h(x, x / \varepsilon)$, is highly oscillating. Our purpose is to study the behaviour of the problem when $\varepsilon$ tends to 0 . In Section 1, we state the homogenized problem in a somewhat general case. Anisotropic effects not only appear on the coefficients but also on the saturation:

Theorem 0.2 There exists a pressure $p_{0}$, two saturation functions $\Xi_{1}, \Xi_{2}$ such that $\left(p_{0}, \Xi_{1}, \Xi_{2}\right)$ is a solution of the homogenized problem:

$$
\left(\mathcal{P}^{\star}\right)\left\{\begin{array}{l}
\text { Find }\left(p_{0}, \Xi_{1}, \Xi_{2}\right) \in V_{a} \times L^{\infty}(\Omega) \times L^{\infty}(\Omega) \text { such that } \\
\int_{\Omega} \mathcal{A} \cdot \nabla p_{0} \nabla \phi=\int_{\Omega} \underline{b}^{0} \nabla \phi, \quad \forall \phi \in V_{0}, \\
p_{0} \geq 0 \quad \text { et } \quad p_{0} \cdot\left(1-\Xi_{i}\right)=0,(i=1,2) \quad \text { a.e. in } \Omega,
\end{array}\right.
$$

with $\mathcal{A}=\left(\begin{array}{cc}a_{11}^{\star} & a_{12}^{\star} \\ a_{21}^{\star} & a_{22}^{\star}\end{array}\right), \underline{b}^{0}=\left(\begin{array}{c}\Xi_{1} b_{1}^{\star} \\ \Xi_{2} b_{2}^{\star}\end{array}\right)$, the coefficients $a_{i j}^{\star}$ and $b_{i}^{\star}$ being given in definition 1.5.

It is obvious that the homogenized problem is not so clear since we are unable to prove that the two saturation functions belong to $[0,1]$ in cavitated areas. However we can prove that the homogenized problem has a particular solution with an isotropic saturation belonging to $[0,1]$, that is:

Theorem $0.3\left(\mathcal{P}^{\star}\right)$ admits at least one solution $\left(p_{0}, \Xi, \Xi\right)$ satisfying $H\left(p_{0}\right) \leq \Xi \leq 1$.

In Section 2, we treat some particular case, i.e. the oblique roughness case, in which two different saturation functions belonging to [0,1] appear. Interestingly, the transverse / longitudinal roughness case is derived from the previous description and appears to be well posed. At last, in Section 3, a numerical simulation illustrates the behaviour of both pressure and saturation when $\varepsilon$ tends to 0 . 


\section{Homogénéisation du modèle d'Elrod-Adams - Cas général}

Hypothèse 1 Soient $Y=] 0,1\left[2\right.$, a et $b$ deux fonctions définies sur $\Omega \times \mathbb{R}^{2}$, appartenant à $L^{2}(\Omega ; C(Y))$ ou $L^{2}(Y ; C(\bar{\Omega})), Y$-périodiques, bornées, supérieures à une constante strictement positive.

Définition 1.1 Soient $a_{\varepsilon}$ et $b_{\varepsilon}$ définies sur $\Omega$ par $a_{\varepsilon}(x)=a\left(x, \frac{x}{\varepsilon}\right)$ et $b_{\varepsilon}(x)=b\left(x, \frac{x}{\varepsilon}\right)$. Soit le problème

$$
\left(\mathcal{P}_{\varepsilon}\right)\left\{\begin{array}{l}
\text { Trouver }\left(p_{\varepsilon}, \theta_{\varepsilon}\right) \in V_{a} \times L^{\infty}(\Omega) \text { tel que } \\
\int_{\Omega} a_{\varepsilon} \nabla p_{\varepsilon} \cdot \nabla v d x=\int_{\Omega} \theta_{\varepsilon} b_{\varepsilon} \frac{\partial v}{\partial x_{1}} d x, \quad \forall v \in V_{0}, \\
p_{\varepsilon} \geq 0, \quad H\left(p_{\varepsilon}\right) \leq \theta_{\varepsilon} \leq 1 \quad \text { p.p.dans } \Omega .
\end{array}\right.
$$

Lemme 1.2 Il existe $\left(p_{0}, p_{1}, \theta_{0}\right) \in V_{a} \times L^{2}\left(\Omega ; H_{\sharp}^{1}(Y) / \mathbb{R}\right) \times L^{2}(\Omega \times Y)$ tel que $\left(p_{\varepsilon}, \nabla p_{\varepsilon}, \theta_{\varepsilon}\right)$ converge double-échelle, à une sous-suite près, vers $\left(p_{0}, \nabla p_{0}+\nabla_{y} p_{1}, \theta_{0}\right)$.

Preuve Les convergences sont issues d'estimations a priori indépendantes de $\varepsilon$ (voir $[1,10,11]$ ), en norme $H^{1}$ pour la pression, en norme $L^{2}$ pour la saturation.

En choisissant des fonctions tests particulières dans le problème $\left(\mathcal{P}_{\varepsilon}\right)($ voir $[1,11])$, on établit :

\section{Proposition 1.3}

- Equation macroscopique : pour tout $\phi$ appartenant à $V_{0}$,

$$
\int_{\Omega}\left(\int_{Y} a\left[\nabla p_{0}+\nabla_{y} p_{1}\right] d y\right) \cdot \nabla \phi d x=\int_{\Omega}\left(\int_{Y} \theta_{0} b d y\right) \frac{\partial \phi}{\partial x_{1}} d x
$$

- Equation microscopique : pour presque tout $x \in \Omega$, pour tout $\psi$ appartenant à $H_{\sharp}^{1}(Y)$ (voir $[1,11]$ pour la définition de $H_{\sharp}^{1}(Y)$ ),

$$
\int_{Y} a\left[\nabla p_{0}+\nabla_{y} p_{1}\right] \cdot \nabla_{y} \psi d y=\int_{Y} \theta_{0} b \frac{\partial \psi}{\partial y_{1}} d y
$$

Définition 1.4 Les problèmes locaux (resp. notés $\left(\mathcal{M}_{i}^{\star}\right),\left(\mathcal{N}_{i}^{\star}\right)$ et $\left.\left(\mathcal{N}_{i}^{0}\right), i=1,2\right)$ sont ainsi définis :

$$
\left\{\begin{array}{l}
\text { Trouver } W_{i}^{\star}, \chi_{i}^{\star}, \chi_{i}^{0} \in L^{2}\left(\Omega ; H_{\sharp}^{1}(Y) / \mathbb{R}\right) \text { tels que, pour presque tout } x \in \Omega \text {, pour tout } \psi \in H_{\sharp}^{1}(Y), \\
\int_{Y} a \nabla_{y} W_{i}^{\star} \nabla_{y} \psi=\int_{Y} a \frac{\partial \psi}{\partial y_{i}}, \quad \int_{Y} a \nabla_{y} \chi_{i}^{\star} \nabla_{y} \psi=\int_{Y} b \frac{\partial \psi}{\partial y_{i}}, \quad \int_{Y} a \nabla_{y} \chi_{i}^{0} \nabla_{y} \psi=\int_{Y} \theta_{0} b \frac{\partial \psi}{\partial y_{i}} .
\end{array}\right.
$$

Définition $1.5 a_{i, j}^{\star}=\widetilde{a} \delta_{i j}-\left[\widetilde{a \frac{\partial W_{j}^{\star}}{\partial y_{i}}}\right], b_{i}^{\star}=\widetilde{b} \delta_{1 i}-\left[\widetilde{a \frac{\partial \chi_{i}^{\star}}{\partial y_{i}}}\right]$, avec $\widetilde{f}(x)=\int_{Y} f(x, y) d y$.

Nous établissons la preuve du théorème 0.2 , qui décrit le problème homogénéisé :

Preuve du théorème $\mathbf{0 . 2}$ On montre, par les techniques d'éclatement périodique [10], à partir des propriétés des solutions $\left(p_{\varepsilon}, \theta_{\varepsilon}\right)$, que $p_{0} \geq 0$ et $H\left(p_{0}\right) \leq \theta_{0} \leq 1$ p.p. dans $\Omega \times Y$. Par ailleurs, le problème local $\left(\mathcal{M}_{i}^{\star}\right)\left(\right.$ resp. $\left.\left(\mathcal{N}_{i}^{\star}\right),\left(\mathcal{N}_{i}^{0}\right)\right)$ admet une unique solution $W_{i}^{\star}\left(\right.$ resp. $\left.\chi_{i}^{\star}, \chi_{i}^{0}\right)$. Par suite, on obtient

$$
p_{1}(x, y)=-\left(\begin{array}{l}
W_{1}^{\star}(x, y) \\
W_{2}^{\star}(x, y)
\end{array}\right) \cdot \nabla p_{0}(x)+\chi_{1}^{0}(x, y), \quad \text { dans } L^{2}\left(\Omega ; H_{\sharp}^{1}(Y) / \mathbb{R}\right) .
$$

Le problème homogénéisé est obtenu en remplaçant $p_{1}$ par l'expression ci-dessus dans l'équation macroscopique (1), et en définissant les quantités

$$
b_{i}^{0}=\widetilde{\left(\theta_{0} b\right)} \delta_{1 i}-\widetilde{\left(a \frac{\partial \chi_{1}^{0}}{\partial y_{i}}\right)}, \quad b_{i}^{\star}=\widetilde{b} \delta_{1 i}-\widetilde{\left(a \frac{\partial \chi_{1}^{\star}}{\partial y_{i}}\right)} \quad \text { et } \quad \Xi_{i}=b_{i}^{0} / b_{i}^{\star} .
$$


Remarque 1 Dans les zones non-cavitées (i.e. $p_{0}>0, \Xi_{i}=1$ ), nous obtenons l'équation de Reynolds homogénéisée classique [6,8]. Cependant:

(a) la propriété $\left(H\left(p_{0}\right) \leq \Xi_{i} \leq 1\right)$ n'est pas garantie, i.e. nous ne savons pas montrer que les fonctions de saturation homogénéisées sont inférieures à 1 dans les zones de cavitation! Nous montrons dans la section 2 qu'il est possible, sous des hypothèses supplémentaires, de dépasser ces difficultés partiellement ou complètement. Par ailleurs, les techniques utilisées pour le problème $(\mathcal{P})$ ne permettent plus d'établir un résultat d'unicité pour $\left(\mathcal{P}^{\star}\right)$,

(b) des algorithmes permettent la résolution numérique du problème $(\mathcal{P})$ (voir [3,7]), mais ne permettent pas de construire une solution de $\left(\mathcal{P}^{\star}\right)$ avec deux fonctions de saturation distinctes.

Le théorème 0.3 établit, parmi les solutions de $\left(\mathcal{P}^{\star}\right)$, l'existence d'une solution $\left(p_{0}, \Xi, \Xi\right)$ satisfaisant la propriété $H\left(p_{0}\right) \leq \Xi \leq 1$ :

Preuve du théorème $\mathbf{0 . 3}$ On introduit un problème pénalisé $\left(\mathcal{P}_{\eta}\right)$, dans lequel la saturation $\theta$ est remplacée par $H_{\eta}(p)$, où $H_{\eta}$ est une approximation du graphe de Heaviside, par exemple

$$
H_{\eta}(z)=\chi_{] \eta,+\infty[}(z)+\frac{z}{\eta} \chi_{[0, \eta]}(z) \text {. }
$$

Le résultat est obtenu par homogénéisation de $\left(\mathcal{P}_{\eta}\right)$, puis passage à la limite sur le paramètre de pénalisation.

\section{Cas particulier : rugosités obliques}

En homogénéisation, la séparation des variables microscopiques permet classiquement d'obtenir des résultats significatifs; l'hypothèse suivante est inspirée de cette remarque.

Hypothèse 2 Soit $\alpha \in \mathbb{R}, a_{\varepsilon}$ et $b_{\varepsilon}$ deux fonctions telles que

$$
\forall x \in \Omega,\left\{\begin{array} { l } 
{ a _ { \varepsilon } ( x ) = a _ { 1 } ( x , \frac { X _ { 1 } ^ { \alpha } ( x ) } { \varepsilon } ) a _ { 2 } ( x , \frac { X _ { 2 } ^ { \alpha } ( x ) } { \varepsilon } ) , } \\
{ b _ { \varepsilon } ( x ) = b _ { 1 } ( x , \frac { X _ { 1 } ^ { \alpha } ( x ) } { \varepsilon } ) b _ { 2 } ( x , \frac { X _ { 2 } ^ { \alpha } ( x ) } { \varepsilon } ) , }
\end{array} \quad \text { avec } \left\{\begin{array}{l}
X_{1}^{\alpha}(x)=\cos \alpha x_{1}+\sin \alpha x_{2}, \\
X_{2}^{\alpha}(x)=-\sin \alpha x_{1}+\cos \alpha x_{2},
\end{array}\right.\right.
$$

les fonctions $a_{i}$ et $b_{i}$ étant bornées, supérieures à une constante strictement positive.

Théorème 2.1 Sous l'hypothèse 2, le problème homogénéisé est ainsi défini :

avec les expressions

$$
\left(\mathcal{P}^{\star}\right)\left\{\begin{array}{l}
\text { Trouver }\left(p_{0}, \Xi_{1}, \Xi_{2}\right) \in V_{a} \times L^{\infty}(\Omega) \times L^{\infty}(\Omega) \text { tel que } \\
\int_{\Omega} \mathcal{A} \cdot \nabla p_{0} \nabla v d x=\int_{\Omega} \underline{b}_{1}^{0} \frac{\partial v}{\partial x_{1}} d x+\int_{\Omega} \underline{b}_{2}^{0} \frac{\partial v}{\partial x_{2}} d x, \quad \forall v \in V_{0}, \\
p_{0} \geq 0, \quad H\left(p_{0}\right) \leq \Xi_{i} \leq 1, \quad(i=1,2) \quad \text { p.p. dans } \Omega
\end{array}\right.
$$

$$
\begin{gathered}
\mathcal{A}(x)=\left(\begin{array}{cc}
a_{1}^{\star}(x) & 0 \\
0 & a_{2}^{\star}(x)
\end{array}\right)+\left(a_{1}^{\star}(x)-a_{2}^{\star}(x)\right) \sin \alpha\left(\begin{array}{r}
-\sin \alpha \cos \alpha \\
\cos \alpha \sin \alpha
\end{array}\right), \\
\underline{b}_{1}^{0}(x)=-\left(b_{1}^{\star}(x) \Xi_{1}(x)-b_{2}^{\star}(x) \Xi_{2}(x)\right) \sin ^{2} \alpha+b_{1}^{\star}(x) \Xi_{1}(x), \\
\underline{b}_{2}^{0}(x)=\left(b_{1}^{\star}(x) \Xi_{1}(x)-b_{2}^{\star}(x) \Xi_{2}(x)\right) \sin \alpha \cos \alpha,
\end{gathered}
$$


et les coefficients homogénéisés $(i, j=1,2$ et $j \neq i): a_{i}^{\star}=\frac{\widetilde{a_{j}}}{\widetilde{a_{i}^{-1}}}$ et $b_{i}^{\star}=\frac{1}{\widetilde{a_{i}^{-1}}} \widetilde{\left(\frac{b}{a_{i}}\right)}$

De plus, $\left(p_{0}, \theta_{0}\right)$ étant la limite double-échelle de $\left(p_{\varepsilon}, \theta_{\varepsilon}\right)$ (solution du problème $\left(\mathcal{P}^{\varepsilon}\right)$ ), le problème $\left(\mathcal{P}^{\star}\right)$ admet une solution $\left(p_{0}, \Xi_{1}, \Xi_{2}\right)$, avec

$$
\Xi_{i}=\frac{1}{\left(\frac{b}{a_{i}}\right)} \widehat{\left(\frac{\theta_{0} b}{a_{i}}\right)}, \quad i=1,2 .
$$

Preuve La démonstration s'effectue en trois étapes : 1- Réécriture du problème $\left(\mathcal{P}_{\varepsilon}\right)$ dans le système de coordonnées $X^{\alpha}=\left(X_{1}^{\alpha}, X_{2}^{\alpha}\right)$, 2- Homogénéisation du problème dans le système de coordonnées $X^{\alpha}$, 3- Réécriture du problème homogénéisé dans le système de coordonnées initial.

Remarque 2 L'hypothèse 2 permet de poser un problème homogénéisé dans lequel apparaissent deux fonctions de saturation distinctes satisfaisant la propriété $H\left(p_{0}\right) \leq \Xi_{i} \leq 1$. Par ailleurs, dans le cas des rugosités transverses et/ou longitudinales $(\alpha=k \pi / 2, k \in \mathbb{Z})$, le problème homogénéisé est un problème de Reynolds généralisé dans lequel n'intervient qu'une seule fonction de saturation et pour lequel on peut obtenir des résultats d'unicité.

Remarque 3 Une démarche initiale "naïve" consisterait à tenter de déterminer une équation limite satisfaite par les limites faibles de $\left(p_{\varepsilon}, \theta_{\varepsilon}\right)$ (en norme $H^{1}$ pour la pression, $L^{2}$ pour la saturation), i.e. $\left(p_{0}, \widetilde{\theta_{0}}\right)$. Or, si la limite faible de la pression intervient effectivement dans l'équation homogénéisée, ce n'est pas la limite faible de la saturation qui joue le rôle de saturation macroscopique : l'équation (3) montre en effet que les saturations limites $\Xi_{i}$ sont des moyennes de $\theta_{0}$ pondérées par l'influence des rugosités, même lorsque le problème est bien posé. Cette équation traduit l'anisotropie sur les fonctions de saturation, et donne un lien explicite entre ces fonctions - macroscopiques - et la saturation microscopique $\theta_{0}$.

Remarque 4 La formulation du problème de la digue pour des configurations géométriques générales, introduite dans [2,9], est similaire à celle du problème de lubrification. Il est possible d'homogénéiser le problème afin de prendre en compte l'influence de couches stratifiées (coefficient de perméabilité variable et oscillant). L'analyse de configurations avec des strates obliques fait également apparaître des phénomènes de double saturation. Dans le cas -plus général- où la perméabilité est décrite par une matrice non diagonale, il est possible d'établir la non-convergence des zones non-saturées [12].

\section{Un exemple de résultat numérique}

Un des enjeux est de savoir si la saturation microscopique $\theta_{0}$ dépend effectivement de la variable $y$. En effet, si ce n'est pas le cas, il est facile de conclure à l'isotropie des saturations homogénéisées (i.e. $\left.\Xi_{1}=\Xi_{2}\right)$. Néanmoins, les simulations numériques indiquent que cette hypothèse ne peut être retenue (voir FIG. 1).

Les simulations de la FIG. 1 correspondent à un régime de fonctionnement réaliste pour un coussinet [7]. On compare la solution homogénéisée avec les solutions associées à des rugosités transverses $(\varepsilon=1 / N=$ $1 / 30,1 / 80$ ). Une coupe est effectuée à $x_{2}$ fixé (afin d'observer les effets d'oscillations dans la direction $x_{1}$ ) et permet d'illustrer la convergence de la pression ainsi que le comportement de la saturation. La convergence forte de la pression dans $L^{2}(\Omega)$ est évidente (l'amplitude des oscillations diminue) et il est 

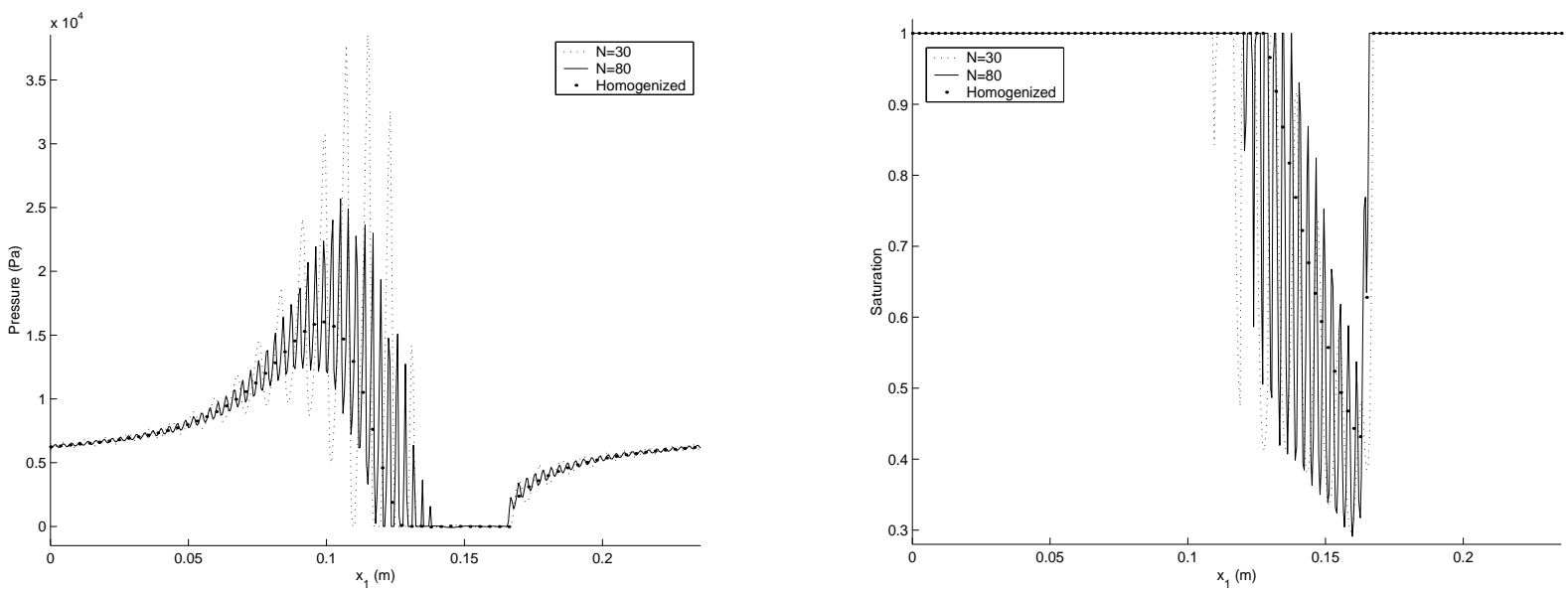

FIG. 1. Pression-saturation - solutions rugueuses et homogénéisée

clair que $\theta_{\varepsilon}$ converge dans $L^{2}(\Omega)$ seulement en un sens faible; en particulier, l'amplitude du gradient explose quand $\varepsilon$ tend vers 0 , indiquant que $\theta_{0}(x, y)$ dépend effectivement de la variable $y$.

\section{Remerciements}

Nous remercions F. Murat pour les fructueuses discussions sur le sujet.

\section{Références}

[1] G. Allaire, Homogenization and two-scale convergence. SIAM J. Math. Anal., 23(6) :1482-1518, 1992.

[2] H.W. Alt, Strömungen durch inhomogene poröse Medien mit freiem Rand. J. Reine Angew. Math., 305 :89-115, 1979.

[3] H.W. Alt, Numerical solution of steady-state porous flow free boundary problems. Numer. Math., 36(1) :73-98, 1980/81.

[4] S.J. Alvarez, R. Oujja, On the uniqueness of the solution of an evolution free boundary problem in theory of lubrication. Nonlinear Anal., 54(5) :845-872, 2003.

[5] S.J. Alvarez, J. Carrillo, A free boundary problem in theory of lubrication. Comm. Partial Differential Equations, 19(11-12) :1743-1761, 1994.

[6] G. Bayada, M. Chambat, Homogenization of the Stokes system in a thin film flow with rapidly varying thickness. RAIRO Modél. Math. Anal. Numér., 23(2) :205-234, 1989.

[7] G. Bayada, M. Chambat, C. Vazquez, Characteristics method for the formulation and computation of a free boundary cavitation problem. J. Comput. Appl. Math., 98(2) :191-212, 1998.

[8] G. Bayada, J.B. Faure, A double-scale analysis approach of the Reynolds roughness. Comments and application to the journal bearing. ASME J. of Tribology, $111: 323-330,1989$.

[9] H. Brezis, D. Kinderlehrer, G. Stampacchia, Sur une nouvelle formulation du problème de l'écoulement à travers une digue. C. R. Acad. Sci. Paris Sér. A-B, 287(9) :A711-714, 1978.

[10] D. Cioranescu, A. Damlamian, G. Griso, Periodic unfolding and homogenization. C. R. Math. Acad. Sci. Paris, 335(1) :99-104, 2002.

[11] D. Lukkassen, G. Nguetseng, P. Wall, Two-scale convergence. Int. J. Pure Appl. Math., 2(1) :35-86, 2002.

[12] J.F. Rodrigues, Some remarks on the homogenization of the dam problem. Manuscripta Math., 46(1-3) :65-82, 1984. 